\title{
Covalent Layer-by-Layer Assemblies of Polyelectrolytes and Homobifunctional Spacer
}

\begin{tabular}{|r|l|}
\hline Journal: & Langmuir \\
\hline Manuscript ID: & la-2010-01670g \\
\hline Manuscript Type: & Article \\
\hline Date Submitted by the & $26-$ Apr-2010 \\
\hline Complete List of Authors: & $\begin{array}{l}\text { El Haitami, Alae; INSERM, UMR 977 } \\
\text { Thomann, Jean-Sebastien; Faculte de Pharmacie, UMR 7199 } \\
\text { CNRS/Université de Strasbourg } \\
\text { Jierry, Loic; CNRS, Institut Charles Sadron } \\
\text { Audrey, Parat; INSERM, UMR 977 } \\
\text { Voegel, Jean-Claude; INSERM, UMR 977 } \\
\text { Schaaf, Pierre; Institut Charles Sadron, CNRS, Université de } \\
\text { Strasbourg } \\
\text { Senger, Bernard; INSERM, U.595 } \\
\text { Boulmedais, Fouzia; Institut Charles Sadron } \\
\text { Frisch, Benoit; Faculte de Pharmacie, UMR 7199 CNRS/Université } \\
\text { de Strasbourg }\end{array}$ \\
\hline
\end{tabular}

\section{scholaroNE \\ Manuscript Central}




\title{
Covalent Layer-by-Layer Assemblies of Polyelectrolytes
}

\section{and Homobifunctional Spacer}

\author{
Alae E. El Haitami ${ }^{1,2,3, \dagger}$, Jean-Sébastien Thomann ${ }^{4, \dagger}$, Lö̈ Jierry ${ }^{3}$, Audrey Parat ${ }^{1,2}$, Jean-Claude \\ Voegel $^{1,2}{ }^{\text {, Pierre Schaaf }}{ }^{3}$, Bernard Senger ${ }^{1,2}$, Fouzia Boulmedais $^{3,} *$ and Benoît Frisch $^{4}$
}

1) Institut National de la Santé et de la Recherche Médicale, UMR 977, 11, rue Humann, 67085

Strasbourg Cedex, France. 2) Université de Strasbourg, Faculté de Chirurgie Dentaire, 1, place de l'Hôpital, 67000 Strasbourg. 3) Centre National de la Recherche Scientifique, Institut Charles Sadron, UPR 22, 23 rue du Loess, BP 84047, 67034 Strasbourg Cedex 2, France. 4) Laboratoire de Conception et Application de Molécules Bioactives, UMR 7199 CNRS/Université de Strasbourg. Faculté de Pharmacie, 74 route du Rhin, 67401 Illkirch Cedex, France.

${ }^{\dagger}$ These authors contributed equally

AUTHOR EMAIL ADDRESS: fouzia.boulmedais@ics-cnrs.unistra.fr

RECEIVED DATE (to be automatically inserted after your manuscript is accepted if required according to the journal that you are submitting your paper to)

TITLE RUNNING HEAD: Covalent polymeric architectures.

CORRESPONDING AUTHOR FOOTNOTE. Phone: +33 (0)3 884141 60; Fax: +33 (0)3 88414099 


\begin{abstract}
The step-by-step buildup of organic films through physical or covalent bonds is usually performed by the alternated adsorption of two types of polymeric chains. Overcompensation of the interacting groups after each deposition step (e.g. charge overcompensation in the case of polyelectrolyte multilayers) allows the buildup process to proceed. This overcompensation is intimately linked to the polymeric nature of the interacting species. We report here another type of film architecture also based on step-by-step construction, but involving the covalent bonding, through Sharpless click reaction, between polyelectrolytes (i.e. polyanions) and neutral bifunctional molecules. The films are built by $\mathrm{Cu}(\mathrm{I})$ catalyzed click reaction of poly(acrylic acid) (PAA) functionalized with ethylene glycol (EG) arms each ending with either an alkyne or an azide group, and bifunctionalized EG spacers ended by either alkyne or azide functions. We prove that these systems lead to the regular buildup of films that cover the whole substrate surface and whose roughness varies as the thickness of the film core. The effects of various parameters on the film buildup are investigated. The grafting density of reactive moieties along the PAA chains has no influence on the thickness increment per bilayer. EG spacers bifunctionalized with alkyne groups reacting with PAA chains functionalized with azide arms give films that grow more rapidly than those obtained with azide functionalized EG spacers and alkyne functionalized PAA chains. The influence of the length of the EG arm (grafted on PAA) and of the EG spacer on the film buildup is also investigated: longer arms or longer spacers lead to larger thickness increments per bilayer, except for very large spacers of $50 \mathrm{EG}$ units for which the thickness is the smallest probably due to a size exclusion effects during the deposition.
\end{abstract}

KEYWORDS: Click chemistry, layer-by-layer, polyelectrolytes, bifunctional spacers, $\mathrm{Cu}(\mathrm{I})$ catalyst 


\section{Introduction}

Step-by-step deposition processes permit the construction of nanometric size films in a highly controlled way. The first example of step-by-step construction of electrostatically interacting molecules concerned polyelectrolytes and bolaform molecules of opposite charges. ${ }^{1}$ The process was rapidly extended to polyelectrolyte multilayers which result from the alternated deposition of polyanions and polycations and which represent certainly the most prominent example of step-by-step film construction. Based on electrostatic interactions, these films are extremely simple to realize with applications ranging from optical coating, ${ }^{2,3}$ selective membrane, ${ }^{4,5}$ anti-corrosion coatings ${ }^{6,7}$ and biofuel cells ${ }^{8}$ up to biologically active coatings. ${ }^{9}$ Unfortunately, polyelectrolyte multilayers are usually mechanically very weak. To circumvent this drawback two strategies have been developed: either cross-linking the polyelectrolytes after film buildup ${ }^{10}$ or using a step-by-step construction process in which the polymers are covalently linked on the substrate at each step of the buildup process. This is done by using two polymers or polyelectrolytes bearing complementary chemical groups "clickable" to react spontaneously to form a covalent bond. Different reactions have been proposed for this purpose, the most widely used being between amine groups and activated esters to form an amide bond, ${ }^{11}$ primary amines and azalactone groups $^{12}$ or azide and alkyne groups to form 1,2,3 triazoles moieties. ${ }^{13,14}$ This latter reaction needs $\mathrm{Cu}(\mathrm{I})$ as catalyst. ${ }^{15}$ Such a buildup process requires that after each deposition step an excess of unreacted groups exists allowing the creation of new covalent bonds during the next deposition step. For an electrostatically driven LbL buildup, this corresponds to charge overcompensation. However, a mechanism involving two reactive groups may not necessarily work. For example, it was found that LbL construction with chitosan bearing $\beta$-cyclodextrin cavities and chitosan bearing adamantyl moieties stopped after 3 or 4 deposition steps, ${ }^{16}$ an effect that might be due to the absence of excess of reacting groups after a few deposition steps.

The interacting groups are usually grafted onto commercially available polymer chains through watersoluble spacers and in particular ethylene glycol (EG) spacers. ${ }^{17}$ Therefore, it should be possible to 
build, in an LbL manner, films involving these modified polymers and spacers ended on both sides by groups that can react with complementary groups present along the polymer chains. Here, we demonstrate that such a buildup process, schematically represented in Scheme 1, is indeed possible. This was a priori by far not obvious because the two ends of the bifunctionalized spacers could react with adsorbed polymers and thereby become unavailable for the next buildup step.

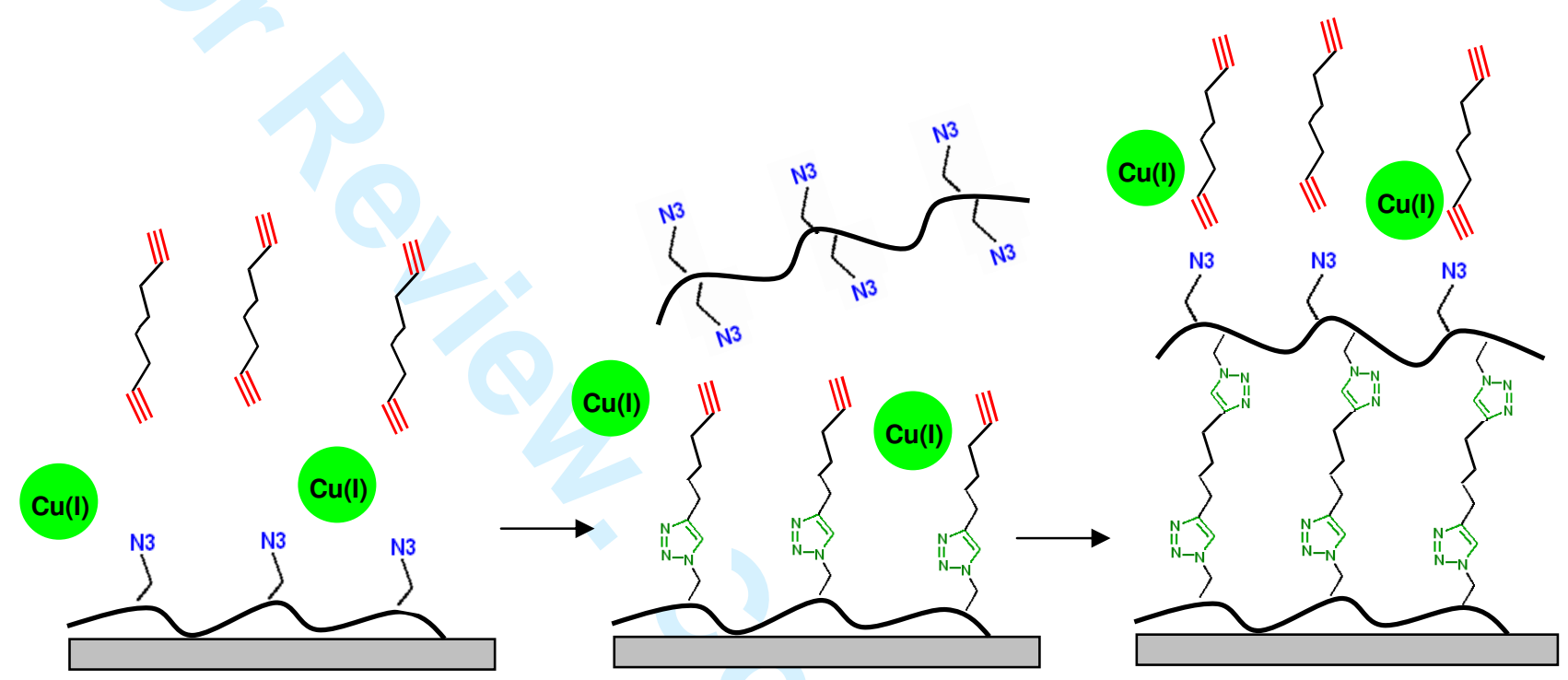

Scheme 1. Schematic representation of the layer-by-layer buildup based on functionalized poly(acrylic acid) (PAA) and a bifunctionalized ethylene glycol (EG) spacer.

Herein, we investigated such systems by using the Sharpless-click reaction between azide and alkyne groups catalyzed by $\mathrm{Cu}(\mathrm{I})$. As a partner of the EG spacers, we selected poly(acrylic acid) (PAA) as the polymer onto which azide or alkyne groups were grafted through EG arms. The buildup of different "polymer/spacer" systems was investigated by quartz crystal microbalance with monitored dissipation (QCM-D) and their morphologies by atomic force microscopy (AFM). The influence of the size of the arms grafted on the PAA, of the size of the EG spacers as well as of the nature of the chemical groups (azide or alkyne) present the polymer and the spacer will be addressed. These systems are, in some respect, the counterpart of the first studied LbL films using charged bolaform molecules and oppositely charged polyelectrolytes. 


\section{Materials and methods}

Chemicals. Polyethyleneimine (PEI, $M=750000$ g/mol, CAS 18 197-8) was purchased from Aldrich and used as received. Poly(acrylic acid) functionalized by ethylene glycol (EG) with either azide groups $\left(\mathrm{PAA}-\mathrm{EG}_{\mathrm{p}}-A z\right)$ or alkyne groups $\left(\mathrm{PAA}-\mathrm{EG}_{\mathrm{p}}-A l k\right)$ at $5 \%$ except otherwise stated and bifunctionalized azide or alkyne EG spacers, $A z-\mathrm{EG}_{\mathrm{q}}-A z$ and $A l k-\mathrm{EG}_{\mathrm{q}}-A l k$, were synthesized as described in Supporting Information (SI). The length of EG oligomers used to graft azide or alkyne on PAA was $p=3$ or 13 . The length of azide bifunctionalized EG spacers was $q=4$ or 13 , whereas it was $q=3$ or 13 when the spacers born two alkyne functional groups. For the sake of simplicity, we will only write $p, q=3$ or 13 for the number of EG repeated units used. Copper(II) sulfate pentahydrate $\left(\mathrm{CuSO}_{4} .5 \mathrm{H}_{2} \mathrm{O}, 99.99 \%\right.$ purity, $M=249.69 \mathrm{~g} / \mathrm{mol}, \mathrm{CAS} 7758-99-8)$ and ascorbic acid sodium salt $\left(\mathrm{C}_{6} \mathrm{H}_{7} \mathrm{NaO}_{6}, \geq 99 \%\right.$ purity, $M=$ $198.11 \mathrm{~g} / \mathrm{mol}$, CAS 134-03-2) were purchased from Aldrich and used as received. All solutions were prepared with ultra pure water (Milli-Q Plus system, Millipore, Billerica, MA). The pH of all solutions was adjusted to 3.5 using $0.1 \mathrm{M} \mathrm{HNO}_{3}$.

Film preparation. The synthesized modified polyanions and bifunctionalized molecules were used for the buildup of $\mathrm{PAA}-\mathrm{EG}_{\mathrm{p}}-A z / A l k-\mathrm{EG}_{\mathrm{q}}-A l k$ and $\mathrm{PAA}-\mathrm{EG}_{\mathrm{p}}-A l k / A z-\mathrm{EG}_{\mathrm{q}}-A z$ films according to the layerby-layer (LbL) technique. After the deposition of a PEI precursor layer, the LbL assembly was performed by alternatively exposing the substrate to $\mathrm{PAA}-\mathrm{EG}_{\mathrm{p}}-A z$ and $A l k-\mathrm{EG}_{\mathrm{q}}-A l k$ (or $\mathrm{PAA}-\mathrm{EG}_{\mathrm{p}}-A l k$ and $\left.A z-\mathrm{EG}_{\mathrm{q}}-A z\right)$ solutions containing copper sulfate and sodium ascorbate for 5 min, with an intermediate rinsing after each polymer and spacer adsorption with a copper sulfate/sodium ascorbate solution. Fresh polymer dipping solutions were made up in a constant volume ratio of 3 (functionalized PAA solution at $0.83 \mathrm{mg} / \mathrm{mL}$ or bifunctionalized spacer solution at $1.66 \mathrm{mg} / \mathrm{mL}): 1\left(\mathrm{CuSO}_{4}\right.$ solution at $0.36 \mathrm{mg} / \mathrm{mL}$ ): 1 (sodium ascorbate stock solution at $8.8 \mathrm{mg} / \mathrm{mL}$ ). Fresh rinsing solutions were made up in a similar ratio, however, replacing the polymer solution by water. The ascorbate stock solution was maintained under nitrogen during the whole buildup to prevent its oxidation. The evolution of the frequencies and dissipations measured by QCM-D during the film buildup showed that 5 min is a 
sufficient time for adsorption and rinsing of both film components (Figure S-1 in SI).

\title{
Quartz crystal microbalance with monitored dissipation (QCM-D) measurements. The QCM-D
}

\author{
experiments were performed on a Q-Sense D 300 apparatus (Q-Sense AB, Göteborg, Sweden) by
} monitoring the resonance frequencies of silica coated crystals, as well as the dissipation factors at four frequencies (the fundamental frequency at $5 \mathrm{MHz}(v=1)$ and the $3 \mathrm{rd}$, 5th, and 7 th harmonics $(v=3,5$ and 7) at 15, 25, and $35 \mathrm{MHz}$ ). The QCM-D results give information on the adsorption process, as well as on viscoelastic properties of the adsorbed film. ${ }^{18}$ In a first approximation, the resonance frequency shifts are proportional to the mass of the film deposited on the crystal per unit area. However, this approximation holds only for homogeneous, quasi-rigid films with not too high the thickness. The reference frequencies and reference dissipations were measured when the crystal was in contact with the rinsing solution. For each film built, the normalized frequency shifts (i.e., the frequency shifts divided by their respective overtone) were practically undistinguishable and the increase of the dissipation was small. The estimation of the mass deposited per unit area can therefore be based on Sauerbrey's relation. Furthermore, assuming a specific mass (arbitrarily chosen to be equal to $1 \mathrm{~g} / \mathrm{cm}^{3}$ ) for the polyelectrolyte film allows converting the mass into the thickness of the film.

Atomic force microscopy (AFM) measurements. The images were obtained in contact mode in liquid conditions with the Nanoscope IV from Veeco (Santa Barbara, CA). The images were carried out with silicon nitride cantilevers, spring constant 0.03 N/m (model MSCTAUHW, Veeco, CA). Several scans were performed over a given surface area. These scans had to give reproducible images to ascertain that there is no sample damage induced by the tip. Deflection and height mode images are scanned simultaneously at a fixed scan rate $(2 \mathrm{~Hz})$ with a resolution of $512 \times 512$ pixels. Data evaluation was performed with the NanoScope software version 5.31r1 (Digital Instruments, Veeco). The buildup of the film was made on a silica coated crystal and the film thickness was measured by using the "scratch" method. Profilometric section analyses of a scratched film allowed us to determine precisely the quality of the film and its thickness over the scanned area. The scratches were achieved with a 
plastic cone tip and were always imaged perpendicular to the fast scan axis. The profiles correspond to a cross section along this axis. The mean thickness of the scratched film was determined by measuring the thickness at least on three areas. For all the observations, the samples were kept under liquid.

\section{Results and discussion}

The buildup of $\left(\mathrm{PAA}-\mathrm{EG}_{\mathrm{p}}-A z / A l k-\mathrm{EG}_{\mathrm{q}}-A l k\right)_{\mathrm{n}}$ and $\left(\mathrm{PAA}-\mathrm{EG}_{\mathrm{p}}-A l k / A z-\mathrm{EG}_{\mathrm{q}}-A z\right)_{\mathrm{n}}$ films with $p$ and $q$ being equal to 3, 13 or 50 was first investigated by QCM-D. We call $n$ the number of "bilayer" deposition steps even if one must keep in mind that certainly the films do not buildup in a well stratified manner and are thus not constituted of consecutive bilayers.
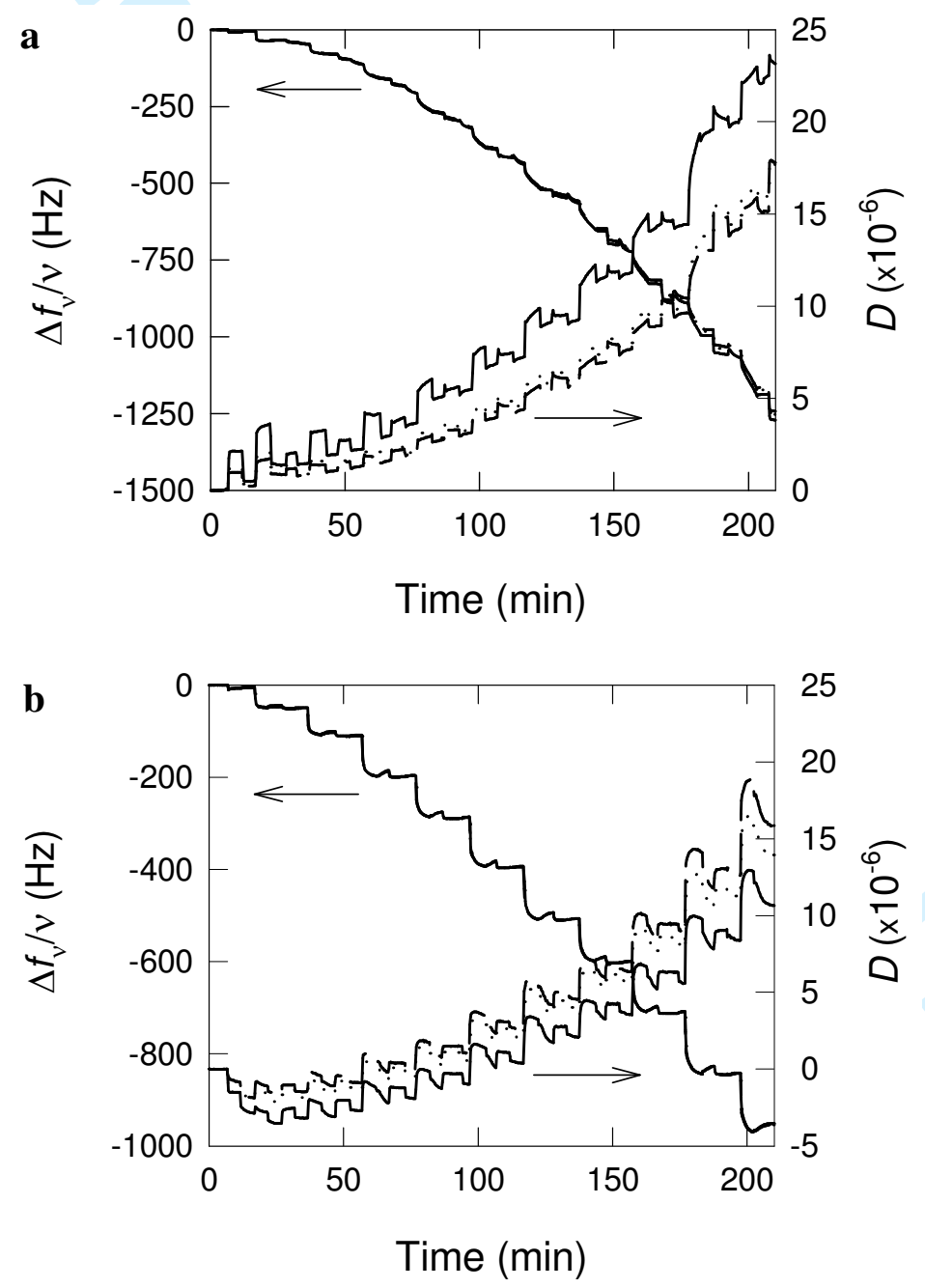

Figure 1. Evolution of the normalized frequency shifts, $\Delta f_{v} / v$, and the dissipation values, $D$, of the third, fifth and seventh overtones as a function of time for (a) PEI-(PAA-EG $\left.{ }_{13}-A z / A l k-\mathrm{EG}_{13}-A l k\right)_{9}-\mathrm{PAA}-\mathrm{EG}_{13}-$ $A z$ and (b) PEI-(PAA-EG $\left.13-A l k / A z-E_{13}-A z\right)_{9}-$ PAA-EG $13-A l k$ measured by QCM-D. 
The constructions were performed at $\mathrm{pH} 3.5$ in the presence of $2.88 \times 10^{-4} \mathrm{~mol} / \mathrm{L}$ of $\mathrm{Cu}(\mathrm{II})$ and $8.88 \times 10^{-4}$ $\mathrm{mol} / \mathrm{L}$ of sodium ascorbate. Both the adsorption and the rinsing steps lasted $5 \mathrm{~min}$, sufficient to observe the stability of the QCM-D signal (Figure S-1 in SI). Figure 1 shows the evolution of the normalized

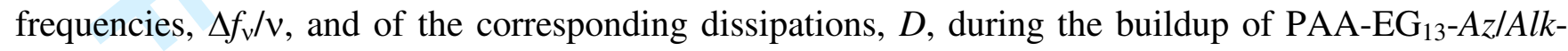
$\mathrm{EG}_{13}-A l k$ and $\mathrm{PAA}-\mathrm{EG}_{13}-A l k / A z-\mathrm{EG}_{13}-A z$. The signals corresponding to the fundamental frequency being often unreliable, we took only the third, fifth and seventh overtones into account. The signals shown in Figure 1 correspond to the two possible cases, namely one where the PAA arms bear azide groups and the other where they bear alkyne groups. In both cases, the films grow regularly. The normalized frequencies corresponding to the overtones $v=3,5$ and 7 are almost indistinguishable one from each other. In addition, the dissipations are of the order of $15 \times 10^{-6}$ which is rather small. For comparison, the typical dissipation of a 15-bilayer poly(sodium 4-styrene sulfonate)/poly(allylamine hydrochloride) (PSS/PAH) multilayer film lies typically between $20 \times 10^{-6}$ and $30 \times 10^{-6}$ and these films are known to behave almost as glasses. ${ }^{19}$ For viscoelastic liquid films such as those constructed using hyaluronic acid (HA) and poly(L-lysine) (PLL), the dissipation is typically of the order of $500 \times 10^{-6}$ after the deposition of only 10 bilayers and these films behave as viscous liquids. ${ }^{20}$ Whereas the Sauerbrey relation $^{21}$ between the mass deposited per unit area and the frequency shift applies reasonably good for PSS/PAH films, it does not apply for HA/PLL multilayers. We thus conclude that for our systems the Sauerbrey relation represents a good approximation for the determination of the deposited mass, and thus for the film thickness if one assumes a given specific mass of the film (e.g. $\left.1 \mathrm{~g} / \mathrm{cm}^{3}\right)$. Yet, one must keep in mind that QCM-D is sensitive to mass including the bound water. In other terms, this technique measures the hydrodynamic film thickness which more or less corresponds to the largest extension of the film. All the studied films will be analyzed within the framework of the Sauerbrey approximation. 

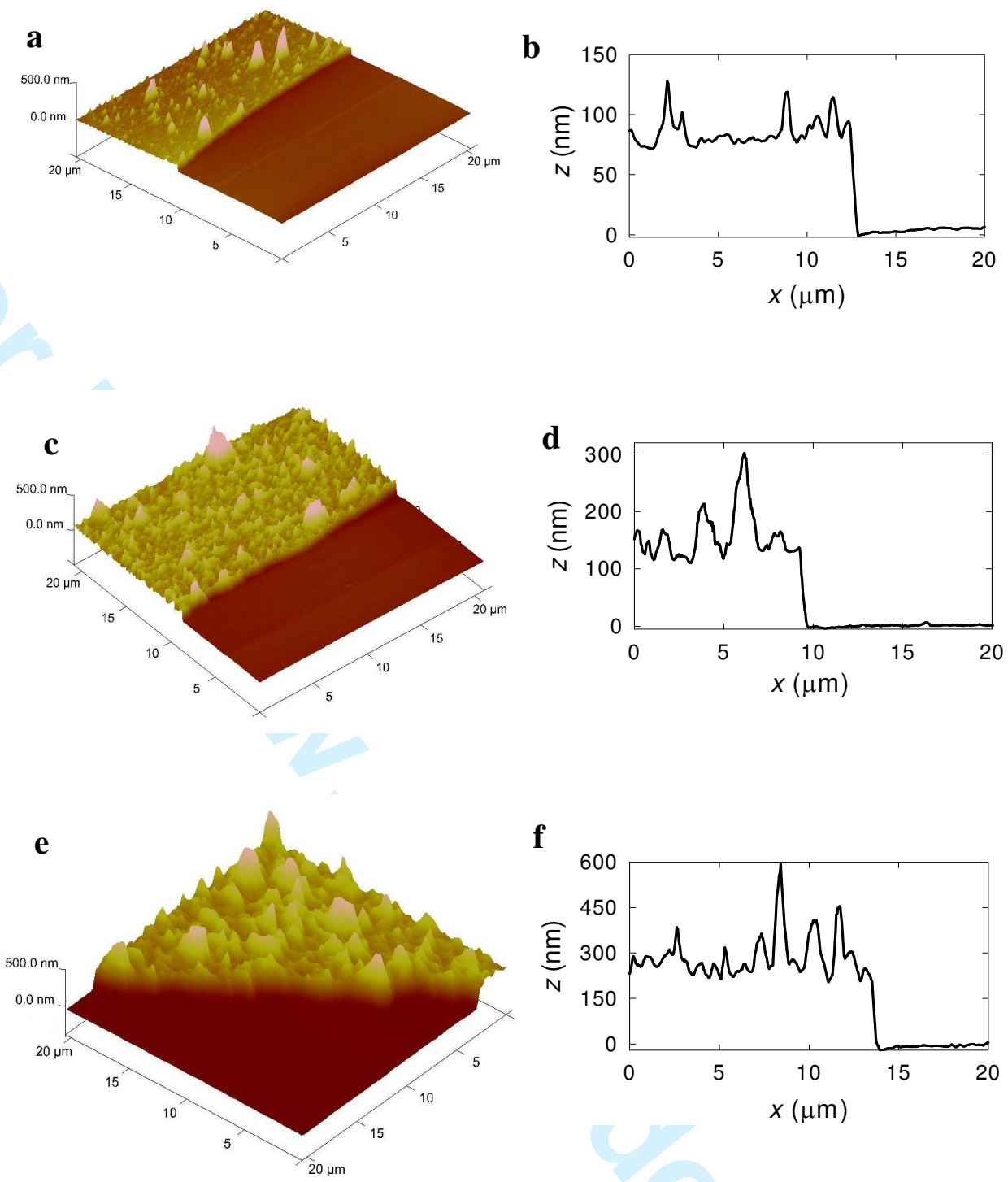

Figure 2. 3D scratched images and profilometric sections $(20 \mu \mathrm{m} \times 20 \mu \mathrm{m})$ obtained in AFM height mode in liquid state of the PEI-(PAA-EG $\left.{ }_{13}-A z / A l k-\mathrm{EG}_{13}-A l k\right)_{\mathrm{n}}-\mathrm{PAA}-\mathrm{EG}_{13}-A z$ film: $(\mathrm{a}, \mathrm{b}) n=4$, (c, d) $n=$ 9 and $(\mathrm{e}, \mathrm{f}) n=14$. The film was built on a QCM-D quartz crystal. The zero value line in $\mathrm{Z}$ is arbitrarily defined by the apparatus.

Next, we concentrated on the structural evolution of the films during the buildup process by means of AFM. For this purpose, we investigated the system PEI-(PAA-EG $\left.{ }_{13}-A z / A l k-E_{13}-A l k\right)_{\mathrm{n}}-\mathrm{PAA}-\mathrm{EG}_{13}-A z$ at different numbers of deposition steps. After buildup, the films were scratched in order to be able to determine their thickness. Figure 2 represents three typical AFM images obtained for $n=4,9$ and 14 . 
With $n=4$, the surface is already entirely covered with a 70-nm thick core studded with large spikes extending over almost $50 \mathrm{~nm}$ and rendering the film rough. As the buildup process goes on, both the core and the roughness increase. Table 1 summarizes the thicknesses of the core measured by AFM and the thicknesses measured by QCM-D on the same films. The larger thickness measured by QCM-D is fully compatible with the roughness of the film which increases its hydrodynamic thickness. Due to the spikes formed on its surface, the films exhibit a significant surface roughness which increases with the number of bilayers. This surface roughness induces a larger hydrodynamic thickness measured by QCMD compared to the thickness of the core determined by AFM (Table 1).

Table 1. Evolution of the thickness of the PEI-(PAA-EG $\left.{ }_{13}-A z / A l k-\mathrm{EG}_{13}-A l k\right)_{\mathrm{n}}-\mathrm{PAA}-\mathrm{EG}_{13}-A z$ film during its growth process and comparison of the various film thickness values obtained by AFM and QCM-D measurements. The errors on the thickness values measured by AFM were obtained from the measurement of three different areas.

\begin{tabular}{cccc}
\hline $\boldsymbol{n}$ & $\boldsymbol{d}_{\mathbf{Q C M}-\mathrm{D}}(\mathbf{n m})$ & $\boldsymbol{d}_{\text {AFM }}(\mathbf{n m})$ & $\mathbf{R M S}(\mathbf{n m})$ \\
\hline 4 & 70 & $70 \pm 5$ & 16.3 \\
9 & 220 & $123 \pm 3$ & 36.7 \\
14 & 390 & $230 \pm 5$ & 57.3 \\
\hline
\end{tabular}

In order to better characterize this roughness, we determined the statistical distribution of the height on the different AFM images (Figure 3). This distribution is taken over an unscratched area of size 20 $\mu \mathrm{m} \times 20 \mu \mathrm{m}$ (discretized into $512 \times 512$ pixels) and the zero in height (i.e. $z=0$ ) is taken at the pixel of lowest altitude identified over this area, corresponding roughly to the upper level of the film core. The most frequent height and the width of the distributions are also given in Table S-1 in SI. As the film buildup goes on, the most frequent height shifts towards higher values and the height distribution broadens. 


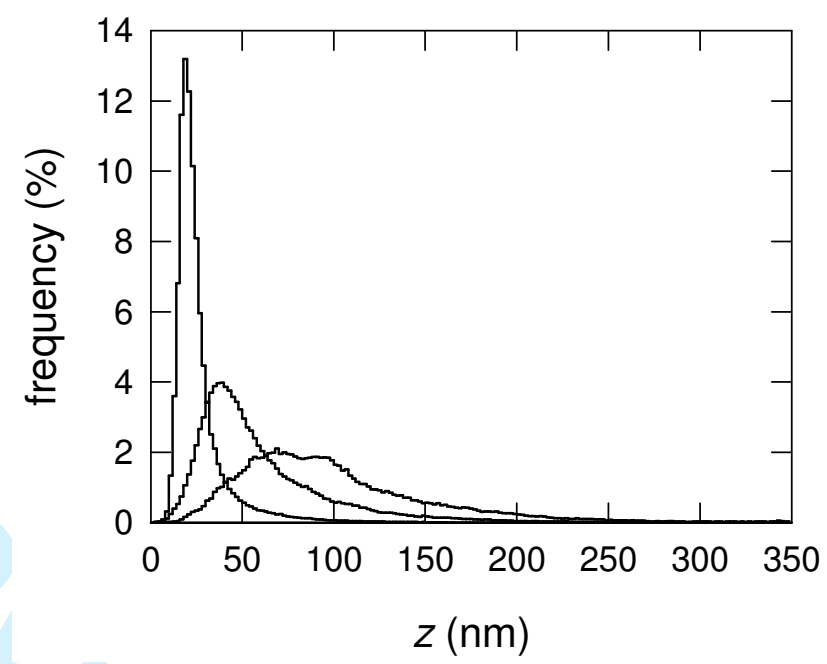

Figure 3: Distribution of the height of the AFM images of PEI-(PAA-EG $\left.13-A z / A l k-\mathrm{EG}_{13}-A l k\right)_{\mathrm{n}}-\mathrm{PAA}-$ $\mathrm{EG}_{13}-A z$ film shown in Fig. 2. From left to right, the peaks correspond to $n=4, n=9$ and $n=14$. Each AFM image is composed by $512 \times 512$ pixels, i.e. each distribution is derived from 262144 values of height. The median, first and third quartiles together with the mean and the standard deviation are summarized in Table S-1 in SI.

Next, we investigated the effects of the nature of the reacting groups, the length of the PAA arms and the length of the linker molecules on the film buildup. The buildups of the different films are summarized in Figure S-2 and S-3. For all these films, the thicknesses were determined after the deposition of 9 bilayers by QCM-D and AFM (Table 2). Let us first concentrate on the effect of the nature of the chemical groups. This is best illustrated in Figure 4 which represents the evolution of the thickness measured by QCM-D during the buildup of PEI-(PAA-EG $\left.13-A z / A l k-\mathrm{EG}_{13}-A l k\right)_{\mathrm{n}}$ and PEI(PAA-EG $\left.{ }_{13}-A l k / A z-\mathrm{EG}_{13}-A z\right)_{\mathrm{n}}$ films. It is apparent that the film grows more rapidly when the alkyne groups are carried by the spacers than by the PAA arms. This is systematically verified for all the investigated films as can be seen in Table 2. 
Table 2. Thicknesses of PEI-(PAA-EG $\left.-A z / A l k-\mathrm{EG}_{\mathrm{q}}-A l k\right)_{9}-\mathrm{PAA}-\mathrm{EG}_{\mathrm{p}}-A z$ and PEI-(PAA-EG $\mathrm{p}-A l k / A z-$ $\left.\mathrm{EG}_{\mathrm{q}}-A z\right)_{9}-\mathrm{PAA}-\mathrm{EG}_{\mathrm{p}}-A l k$ films measured by AFM and QCM-D for short and long EG spacers and PAA arms, corresponding respectively to 3 and 13 repeated EG units. The error bars represent the standard deviation of three independent experiments for QCM-D. For AFM, the thickness was obtained by scratching the film and measuring the height of the scratch on three different areas.

\begin{tabular}{|c|c|c|c|}
\hline $\begin{array}{l}\text { Values of } p \text { in } \\
\text { PAA-EG }-A z\end{array}$ & $\begin{array}{l}\text { Values of } q \text { in } \\
A l k-\mathrm{EG}_{\mathrm{q}}-A l k\end{array}$ & $\begin{array}{c}\text { Thicknesses measured } \\
\text { by QCM-D (nm) }\end{array}$ & $\begin{array}{c}\text { Thicknesses measured } \\
\text { by AFM (nm) }\end{array}$ \\
\hline 3 & 3 & $106 \pm 10$ & $30 \pm 2$ \\
\hline 3 & 13 & $145 \pm 14$ & $119 \pm 3$ \\
\hline 13 & 3 & $121 \pm 9$ & $74 \pm 2$ \\
\hline 13 & 13 & $207 \pm 4$ & $123 \pm 3$ \\
\hline $\begin{array}{l}\text { Values of } p \text { in } \\
\text { PAA-EG }{ }_{p}-A l k\end{array}$ & $\begin{array}{c}\text { Values of } q \text { in } \\
A z-\mathrm{EG}_{\mathrm{q}}-A z\end{array}$ & $\begin{array}{c}\text { Thicknesses measured } \\
\text { by QCM-D (nm) }\end{array}$ & $\begin{array}{c}\text { Thicknesses measured } \\
\text { by AFM (nm) }\end{array}$ \\
\hline 3 & 3 & $17 \pm 3$ & $13 \pm 2$ \\
\hline 3 & 13 & $52 \pm 2$ & $27 \pm 5$ \\
\hline 13 & 3 & $105 \pm 12$ & $60 \pm 7$ \\
\hline 13 & 13 & $140 \pm 40$ & $94 \pm 5$ \\
\hline
\end{tabular}

The differences are particularly striking when the alkyne groups are coupled to the PAA through a short, $p=3$, EG arm where we observe $27 \mathrm{~nm}$ for the system (PAA-EG $\left.3-A l k / A z-\mathrm{EG}_{13}-A z\right)_{9}$ compared to $119 \mathrm{~nm}$ measured for the system (PAA-EG $\left.3-A z / A l k-\mathrm{EG}_{13}-A l k\right)_{9}$ as measured by means of AFM. This effect is explained by the formation of a transient and stable positively charged $\mathrm{Cu}(\mathrm{I}) /$ alkyne complex during the click reaction. It has been demonstrated recently that the formation of this complex has a strong influence of the multilayer buildup process. It favors, for example, the film buildup from neutral polymers bearing alkyne groups, that become positively charged in the presence of $\mathrm{Cu}(\mathrm{I})$ and negatively charged PAA chains bearing azide groups. ${ }^{22}$ When the alkyne groups are borne by the bifunctional spacers, in the presence of $\mathrm{Cu}(\mathrm{I})$, they behave as positive molecules and are thus electrostatically attracted by the PAA chains (if the $\mathrm{pH}$ is high enough). The alkyne groups have thus a higher probability to be in the vicinity of the azide groups and to react than in the inverse case. In contrary, when 
the alkyne groups are worn by the PAA chains, due to electrostatic interactions between the cationic alkyne/Cu(I) complex and the negatively charged carboxylic groups, they are less accessible to the azide groups and the reaction is thus slowed down. This effect must be particularly pronounced for alkyne groups linked to the PAA backbone by short arms, as indeed observed (Table 2). AFM images show that in all cases the films are rough and constituted of a core and spikes (Figure S-4 and S-5 in SI) as the PAA-EG $13-A z / \mathrm{PAA}-\mathrm{EG}_{13}-A l k$ film but with a smaller roughness (Figure S-6 and S-7 in SI).

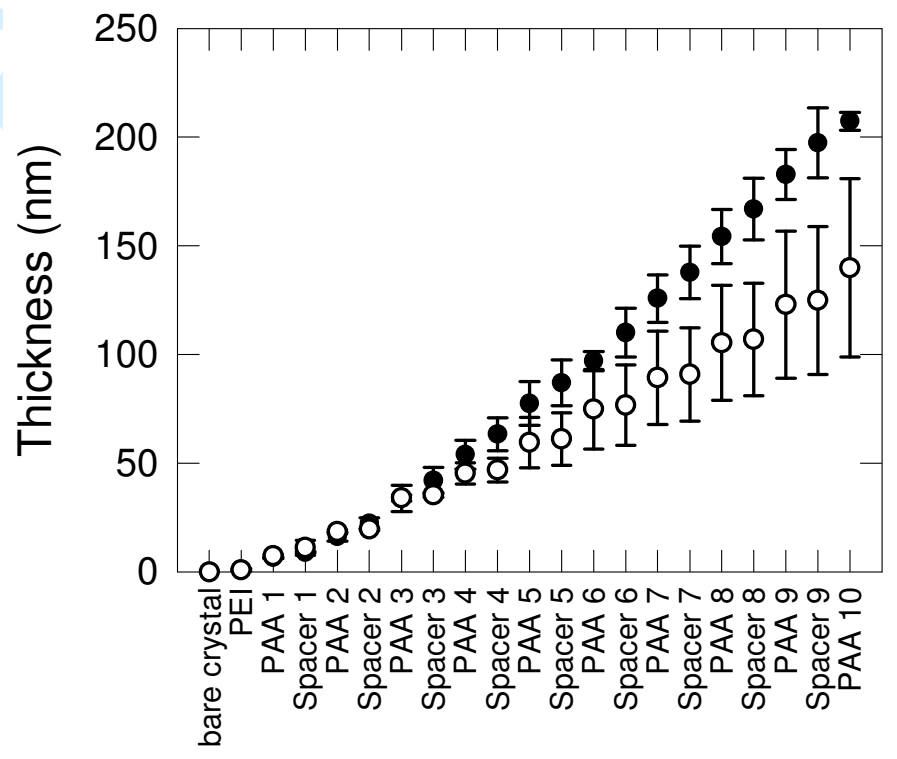

Last layer deposited

Figure 4. Evolution of the thickness of PEI-(PAA-EG $\left.{ }_{13}-A z / A l k-E_{13}-A l k\right)_{9}-\mathrm{PAA}-\mathrm{EG}_{13}-A z(\bigcirc)$ and PEI$\left(\mathrm{PAA}-\mathrm{EG}_{13}-A l k / A z-\mathrm{EG}_{13}-A z\right)_{9}-\mathrm{PAA}-\mathrm{EG}_{13}-A l k(\mathrm{O})$ film as a function of adsorbed layer. The thickness is derived from the frequency shift (for $v=3$ ), measured by QCM-D, using Sauerbrey's relation. The error bars represent the standard deviation of three independent experiments.

As far as the influence of the size of the arms and of the spacers is concerned (when $p, q=3$ or 13), for a given chemical group, longer spacers and longer arms lead, as anticipated, to thicker films. These films present a roughness that is also dependent on the spacer and arm length. Table 3 summarizes the roughness (RMS) measured on AFM images of the different films studied at 9.5 bilayers (Figure S-8 and S-9 in SI). The thickness and roughness effect is enhanced with alkyne bifunctional spacers as can 
be seen in Table 2, Table 3 and Figure 5.

Table 3. Roughness (in $\mathrm{nm}$ ) expressed by the standard deviation of the heights of PEI-(PAA-EG $\mathrm{p}^{-}$ $\left.A z / A l k-\mathrm{EG}_{\mathrm{q}}-A l k\right)_{9}-\mathrm{PAA}-\mathrm{EG}_{\mathrm{q}}-A z$ and PEI-(PAA-EG $\left.{ }_{\mathrm{p}}-A z / A l k-\mathrm{EG}_{\mathrm{q}}-A l k\right)_{9}-\mathrm{PAA}-\mathrm{EG}_{\mathrm{p}}-A z$ films measured on AFM images. Note that this estimation of the roughness is identical with the roughness estimation commonly called RMS.

\begin{tabular}{|c|c|c|c|c|c|}
\hline & $A l k-\mathbf{E G}_{2}-A l k$ & $A l k-\mathbf{E G}_{13}-A l k$ & & $A z-\mathrm{EG}_{2}-A z$ & $A z-\mathrm{EG}_{13}-A z$ \\
\hline PAA-EG $\mathbf{E}_{3}-\boldsymbol{A} z$ & 9.9 & 45.1 & PAA-EG ${ }_{3}-A l k$ & 2.9 & 3.3 \\
\hline PAA-EG $_{13}-A z$ & 11.5 & 36.7 & PAA-EG ${ }_{13}-A l k$ & 3.1 & 7.7 \\
\hline
\end{tabular}

To enrich the study of the EG chain length incidence, we compared the buildup of films based on PAA-EG ${ }_{13}-A l k$ and $A z-\mathrm{EG}_{\mathrm{q}}-A z$ for three different lengths of the bifunctionalized spacer, namely $A z-\mathrm{EG}_{3^{-}}$ $A z, A z-\mathrm{EG}_{13}-A z$ and $A z-\mathrm{EG}_{50}-A z$ (Figure S-10 in SI). By increasing the length of the spacer, we can expect a larger mass increase per bilayer due to the mass of the spacer. This is observed by increasing the length from $\mathrm{EG}_{3}$ to $\mathrm{EG}_{13}$. In contrary, when the length of the spacer is increased from $\mathrm{EG}_{13}$ to $\mathrm{EG}_{50}$, the film grows less. This could be due to the fact that $\mathrm{EG}_{50}$ is long enough to behave as a polymeric chain which adopts, in solution, a coiled conformation and on the surface a mushroom conformation. Steric hindrance then prevents rapidly interactions of the chains from the solution with the surface and thus the buildup of a dense layer. The thickness of $\mathrm{PAA}-\mathrm{EG}_{13}-A l k / A z-\mathrm{EG}_{50}-A z$ amounts to $45 \mathrm{~nm}$ and 57 nm as measured by means of AFM (Figure S-11 in SI) and QCM, respectively. 

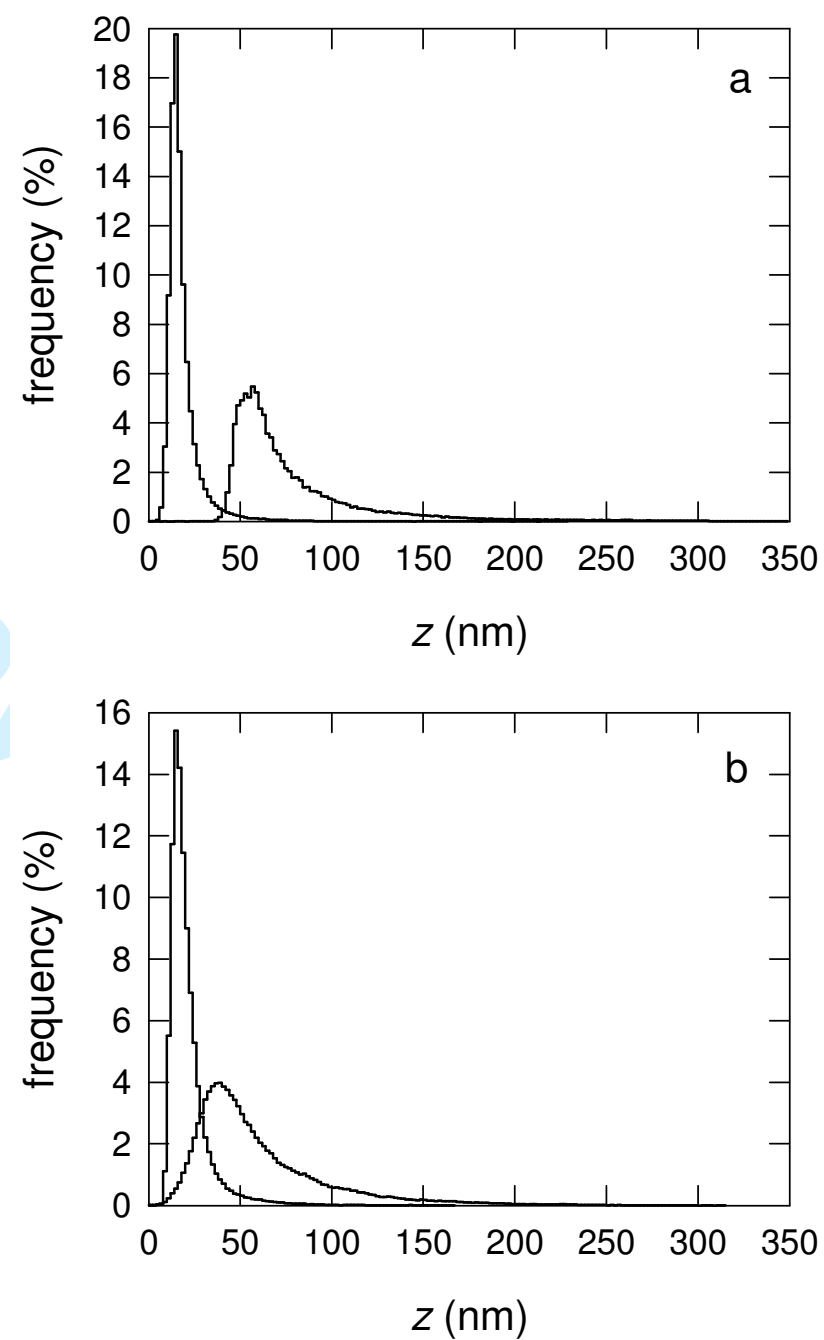

Figure 5. Distribution of the height of the AFM images of (a) PEI-(PAA-EG $\left.-A z / A l k-\mathrm{EG}_{3}-A l k\right)_{9}-\mathrm{PAA}-$ $\mathrm{EG}_{3}-A z$ (left peak) compared to PEI-(PAA-EG $\left.3-A z / A l k-\mathrm{EG}_{13}-A l k\right)_{9}-\mathrm{PAA}-\mathrm{EG}_{3}-A z$ (right peak); (b) PEI(PAA-EG $\left.{ }_{13}-A z / A l k-\mathrm{EG}_{3}-A l k\right)_{9}-\mathrm{PAA}-\mathrm{EG}_{13}-A z$ (left peak) compared to PEI-(PAA-EG ${ }_{13}-A z / A l k-\mathrm{EG}_{13}-$ $A l k)_{9}-\mathrm{PAA}-\mathrm{EG}_{13}-A z$ (right peak).

As already stated, it is not equivalent to have a PAA arm with 13 EG units and a spacer with 3 EG units or vice versa, the difference being enhanced with azide linkers. AFM imaging reveals that for all investigated films the surface is totally covered after deposition of 9 bilayers (Figure S-4 and S-5 in SI). A film is generally constituted of a core covered by spikes whose number by unit area and height depend upon the system. There are almost no spikes on the $\mathrm{PAA}-\mathrm{EG}_{3}-A l k / A z-\mathrm{EG}_{13}-A z$ film whereas the PAA$\mathrm{EG}_{3}-A z / A l k-\mathrm{EG}_{13}-A l k$ film is fully covered with spikes (Figure 6). It can also be pointed out that 
the error bars in Figure 4 are larger for the system with azide spacers than for that with alkyne spacers. As to now, we have no explanation for this observation.

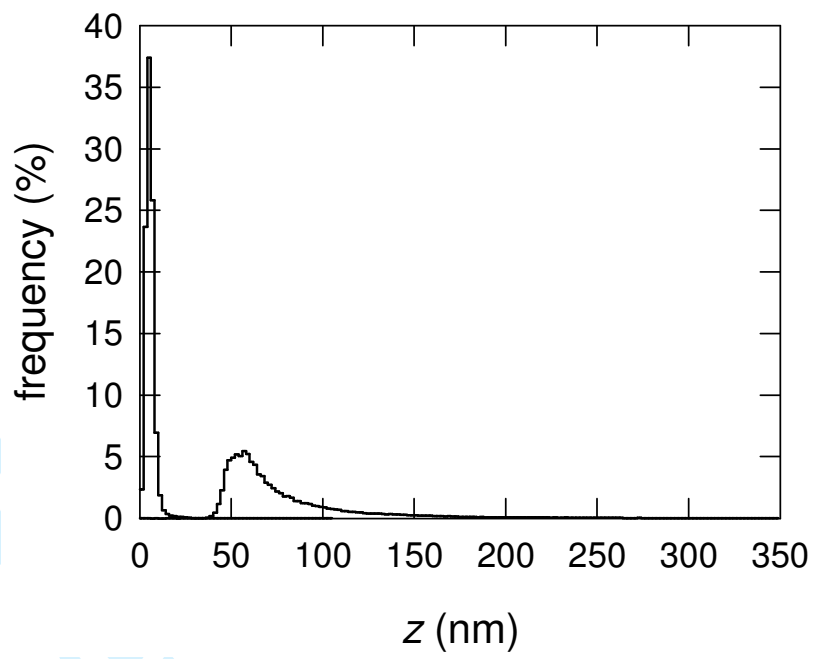

Figure 6. Distribution of the height of the AFM images of PEI-(PAA-EG $\left.-A l k / A z-\mathrm{EG}_{13}-A z\right)_{9}-\mathrm{PAA}-\mathrm{EG}_{3}-$ Alk (left peak) compared to PEI-(PAA-EG $\left.3-A z / A l k-\mathrm{EG}_{13}-A l k\right)_{9}-\mathrm{PAA}_{3}-\mathrm{EG}_{3}-A z$ (right peak).

Finally, we also investigated the effect of the grafting ratio of the PAA chains. We compared the buildup of (PAA-EG $\left.{ }_{3}-A z / A l k-\mathrm{EG}_{3}-A l k\right)_{\mathrm{n}}$ films for grafting ratios of $5 \%, 9 \%$ and $31 \%$ of the PAA chains (Figure S-12 in SI). Both films were built at $\mathrm{pH} 4.4$ because of the insolubility of PAA functionalized at $31 \%$ at lower $\mathrm{pH}$. After the deposition of 5 bilayers on a QCM-D crystal, the thicknesses of the films of $5 \%, 9 \%$ and $31 \%$ grafting ratio were evaluated respectively at 98, 83 and $107 \mathrm{~nm}$ by QCM-D according to the Sauerbrey's relation and respectively at 127,95 , and $111 \mathrm{~nm}$ according to the Voinova model. ${ }^{18}$ The grafting ratio seems thus to have no significant effect on the film thickness, in a first approximation. It may, however, be noted that the ratio of the Sauerbrey thickness to the Voinova thickness equals respectively $0.77,0.87$ and 0.96 . This evolution suggests that the largest grafting ratio might favor the formation of the most rigid film (i.e. with the least dissipation of the vibration energy). Supplementary experiments would be needed to verify this hypothesis but this extension is beyond the scope of this article. 


\section{Conclusion}

We have investigated the step-by-step covalent buildup of polyelectrolyte chains with bifunctional spacers that are bolaform molecules. We used the Sharpless click reaction catalyzed with $\mathrm{Cu}(\mathrm{I})$ between azide and alkyne groups to form the covalent bonds. We proved that such a step-by-step construction is possible and leads to films which rapidly cover the whole surface. The films are, however, rather rough. They are constituted of a core covered by spikes. We found that, for the investigated system, namely $\mathrm{EG}_{\mathrm{q}}$ spacers and PAA chains onto which are grafted $\mathrm{EG}_{\mathrm{p}}-A z$ or $\mathrm{EG}_{\mathrm{p}}-A l k$ arms, it is not equivalent to build the film with a short arm and a long spacer or the contrary. Yet, we found that the longer the arms and the longer the spacers, the higher is the film thickness for a given number of deposition steps except for the spacers of $50 \mathrm{EG}$ units where the thickness is the smallest probably due its steric hindrance preventing interactions of the chains from the solution with the surface.

Acknowledgments. A. E. E. H. thanks the Ministère de l'Enseignement Supérieur et de la Recherche for financial support as well as Philippe Lavalle for his valuable advices in AFM imaging. J.-S. T. is indebted to the Université de Strasbourg for their fellowships. This work was supported by the ANR ClickMultilayer ANR-07-BLAN-0169.

Supporting Information Available. Detailed synthesis of functionalized poly(acrylic acid) (PAA) and bifunctionalized ethylene glycol (EG) spacers by azide or alkyne functions, Buildup of PAA-EG 13- $^{-}$ Az/Alk-EG $\mathrm{EG}_{13}$-Alk film followed by QCM-D, characteristic parameters obtained from the distribution of the height of the film surface analyzed on the AFM images of PAA-EG ${ }_{13}-A z / A l k-\mathrm{EG}_{13}-A l k$ at different steps, Evolution of the film thickness built with $\mathrm{PAA}-\mathrm{EG}_{\mathrm{p}}-A z$ and $A l k-\mathrm{EG}_{\mathrm{q}}-A l k$ according to the length of the EG chain, Evolution of the film thickness built with $\mathrm{PAA}-\mathrm{EG}_{\mathrm{p}}-A l k$ and $A z-\mathrm{EG}_{\mathrm{q}}-A z$ according to the length of the EG chain, AFM height mode scratched images in liquid state of various films accompanied with their profilometric sections for film thickness measurements, Buildup of PAA-EG ${ }_{13^{-}}$ 
$A z /$ PAA-EG ${ }_{13}-A l k$ film followed by QCM-D, AFM height mode images of PAA-EG $\mathrm{p}_{\mathrm{p}}-A z$ and $A l k-\mathrm{EG}_{\mathrm{q}}{ }^{-}$

Alk according to the length of the EG chain, AFM height mode images of PAA-EG $\mathrm{p}-A l k$ and $A z-\mathrm{EG}_{\mathrm{q}}-A z$ according to the length of the EG chain, Buildup of $\mathrm{PAA}-\mathrm{EG}_{13}-A z / A l k-\mathrm{EG}_{3}-A l k, \mathrm{PAA}-\mathrm{EG}_{13}-A z / A l k-$ $\mathrm{EG}_{13}-A l k$ and PAA-EG ${ }_{13}-A l k / A z-\mathrm{EG}_{50}-A z$ films followed by QCM-D, Buildup of PAA-EG $-A z / A l k-\mathrm{EG}_{3}-$ Alk films with 5\%, 9\% and 31\% of functionality followed by QCM-D.

\section{References}

(1) Decher, G.; Hong, J. D. Makromol. Chem., Macromol. Symp 1991, 46, 321-327.

(2) Hiller, J.; Mendelsohn, J. D.; Rubner, M. F. Nat. Mater. 2002, 1, 59-63.

(3) Podsiadlo, P.; Sui, L.; Elkasabi, Y.; Burgardt, P.; Lee, J.; Miryala, A.; Kusumaatmaja, W.; Carman, M. R.; Shtein, M.; Kieffer, J.; Lahann, J.; Kotov, N. A. Langmuir 2007, 23, 7901-7906.

(4) Rmaile, H. H.; Schlenoff, J. B. J. Am. Chem. Soc. 2003, 125, 6602-6603.

(5) Liu, X.; Bruening, M. L. Chem. Mater. 2004, 16, 351-357.

(6) Andreeva, D. V.; Fix, D.; Möhwald, H.; Shchukin, D. G. J. Mater. Chem. 2008, 18, 1738-1740.

(7) Andreeva, D. V.; Fix, D.; Möhwald, H.; Shchukin, D. G. Adv. Mater. 2008, 20, 2789-2794.

(8) Farhat, T. R.; Hammond, P. T. Adv. Funct. Mater. 2005, 15, 945-954.

(9) Chluba, J.; Voegel, J.-C.; Decher, G.; Erbacher, P.; Schaaf, P.; Ogier, J. Biomacromolecules 2001, 2 , 800-805.

(10) Schneider, A.; Vodouhê, C.; Richert, L.; Francius, G.; Le Guen, E.; Schaaf, P.; Voegel, J.-C.; Frisch, B.; Picart, C. Biomacromolecules 2007, 8, 139-145.

(11) Seo, J.; Schattling, P.; Lang, T.; Jochum, F.; Nilles, K.; Theato, P.; Char, K. Langmuir 2010, 26, 1830-1836.

(12) Buck, M. E.; Fhang, J.; Lynn, D. M. Adv. Mater. 2007, 19, 3951-3955.

(13) Bergbreiter, D. E.; Chance, B. S. Macromolecules 2007, 40, 5337-5343.

(14) Such, G. K.; Quinn, J. F.; Quinn, A.; Tjipto, E.; Caruso, F. J. Am. Chem. Soc. 2006, 128, 93189319.

(15) Rostovtsev, V. V.; Green, L. G.; Fokin, V. V.; K.B., S. Angew. Chem. Int. Ed. 2002, 41, 25962599.

(16) Van der Heyden, A.; Wilczewski, M.; Labbé, P.; Auzély, R. Chem. Commun. 2006, 3220-3222.

(17) Rydzek, G.; Thomann, J.-S.; Ben Ameur, N.; Jierry, L.; Mésini, P.; Ponche, A.; Contal, C.; El Haitami, A. E.; Voegel, J.-C.; Senger, B.; Schaaf, P.; Frisch, B.; Boulmedais, F. Langmuir 2009, 26, $2816-2824$.

(18) Voinova, M. V.; Rodahl, M.; Jonson, M.; Kasemo, B. Phys. Scr. 1999, 59, 391-396.

(19) Mertz, D.; Hemmerlé, J.; Boulmedais, F.; Voegel, J.-C.; Lavalle, P.; Schaaf, P. Soft Matter 2007, 3, $1413-1420$.

(20) Collin, D.; Lavalle, P.; Méndez Garza, J.; Voegel, J.-C.; Schaaf, P.; Martinoty, P. Macromolecules 2004, 37, 10195-10198.

(21) Sauerbrey, G. Z. Phys. 1959, 155, 206-222.

(22) Jierry, L.; Ben Ameur, J.; Thomann, J. S.; Frisch, B.; Gonthier, E.; Voegel, J.-C.; Senger, B.; Decher, G.; Félix, O.; Schaaf, P.; Mésini, P.; Boulmedais, F. Macromolecules 2010, 43, 3994-3997. 
1

\section{SYNOPSIS TOC}

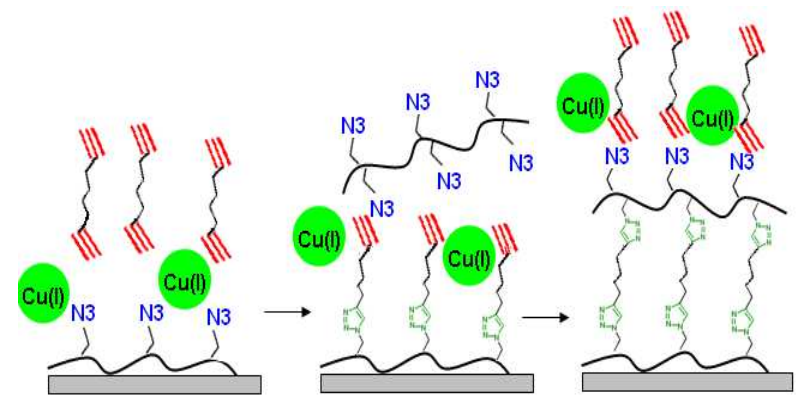

We report a new and original covalent layer-by-layer buildup of films from functionalized polyelectrolytes and bifunctionalized spacers based on the Sharpless click reaction. 